\title{
Fatores relacionados à escolha de métodos contraceptivos na adolescência: uma revisão de literatura
}

\author{
Related factors to the choice of contraceptive methods in adolescence: a literature review \\ Factores relacionados con la elección de métodos anticonceptivos en la adolescencia: \\ una revisión de la literatura
}

Amanda Rodrigues Rios ${ }^{1 *}$, Amanda Duarte de Sena², Bruna Reis Krug ${ }^{3}$, Ellen Karoliny de Oliveira Dantas ${ }^{4}$, Erlen Cristina Botelho Ferronato ${ }^{5}$, Julia Quintiliano Bomfim ${ }^{6}$, Letícia Almeida de Oliveira ${ }^{7}$, Pauline Christina Campos Martins Ferreira ${ }^{8}$, Vinícius Gustavo de Carvalho Moura ${ }^{8}$, Raquel Meirelles Gaspar Coelho Guimarães ${ }^{9}$.

\section{RESUMO}

Objetivo: Identificar os fatores relacionados à escolha do método contraceptivos na adolescência, os riscos e benefícios dos métodos contraceptivos e suas indicações. Revisão bibliográfica: Anticoncepcionais orais podem induzir alterações comportamentais, cerebrais, ósseas e complicações cardiovasculares. Podem ainda servir de proteção em diversas patologias, auxiliar na regulação do ciclo menstrual e oleosidade dérmica. Os dispositivos e sistemas intrauterinos podem alterar padrão menstrual, têm baixo risco de perfuração uterina, taxa de sucesso de $96 \%$ na primeira tentativa de colocação, são seguros e dispensam intervenção diária. Já os contraceptivos hormonais injetáveis são eficientes para casos de dificuldade de adesão diária ao tratamento oral, no entanto podem apresentar ganho de peso e perda de densidade óssea significativa, sendo discutível o uso na puberdade. Implantes subdérmicos de etonogestrel são indicados para reduzir dismenorreia e em casos suscetíveis ao tromboembolismo venoso. Os contraceptivos de barreira são eficazes na prevenção à gravidez e, alguns, também às infecções sexualmente transmissíveis. Considerações finais: Cada paciente deve ser avaliada conforme particularidades clínicas, sociais, pessoais e psicológicas, sendo a escolha do método contraceptivo feita entre médico e paciente. Faz-se necessário expor os riscos e benefícios do método escolhido e orientar o uso associado ao contraceptivo de barreira.

Palavras-chave: Adolescente, Anticoncepcionais, Adesão à medicação.

\begin{abstract}
Objective: Identify factors related to the choice of contraceptive methods in adolescence, the risks and benefits of contraceptive methods and their indications. Bibliographic review: Oral contraceptives can induce behavioral, brain, bone and cardiovascular complications, protect against pathologies, regulate menstrual cycle and dermal oiliness. Intrauterine devices/systems may alter menstrual pattern, have low risk of uterine perforation, high success rate for placement, with failures and expulsions regardless of age, type of device or parity; It is safe, dispenses daily intervention. Injectable hormonal contraceptives are effective for cases of daily oral treatment difficulty. They may present weight gain and significant bone density loss, and the use at puberty is debatable. Subdermal ethnogestrel implants are indicated to reduce dysmenorrhea and in cases susceptible to venous thromboembolism; May generate menstrual unpredictability. Barrier contraceptives prevent pregnancy and sexually transmitted infections. Final considerations: Each patient should be evaluated according to clinical, social, personal and psychological particularities, the choice of contraceptive method made between doctor and patient. It is necessary to expose the risks and benefits of the chosen method and guide the use associated with barrier contraceptives.
\end{abstract}

Keywords: Adolescent, Contraceptive agents, Medication adherence.

\footnotetext{
${ }^{1}$ Centro Universitário de Caratinga (UNEC), Caratinga - MG. *E-mail: amandarios17@hotmail.com

${ }^{2}$ Universidade Nove de Julho (UNINOVE), São Bernardo do Campo - SP.

${ }^{3}$ Universidade Luterana do Brasil (Ulbra), Canoas - RS.

${ }^{4}$ Centro Universitário do Espírito Santo (UNESC), Colatina - ES.

${ }^{5}$ Centro Universitário Ingá (Uningá), Maringá - PR.

${ }^{6}$ Centro Universitário Cesmac (Cesmac), Maceió - AL.

${ }^{7}$ Centro Universitário de Rio Verde (UniRV), Aparecida de Goiânia - GO.

${ }^{8}$ Universidade Federal de Ouro Preto (UFOP), Ouro Preto - MG.

${ }^{9}$ Universidade da Região de Joinville (Univille), Joinville - SC.
} 


\section{RESUMEN}

Objetivo: Identificar factores relacionados con la elección del método anticonceptivo en la adolescencia, los riesgos y beneficios de los métodos anticonceptivos y sus indicaciones. Revisión bibliográfica: Los anticonceptivos orales pueden inducir complicaciones conductuales, cerebrales, óseas y cardiovasculares. También pueden servir como protección en diversas patologías, ayudar en la regulación del ciclo menstrual y la untuosidad dérmica. Los dispositivos y sistemas intrauterinos pueden cambiar los patrones menstruales, tienen un riesgo bajo de perforación uterina, tienen una tasa de éxito del $96 \%$ en el primer intento de colocación, son seguros y no requieren una intervención diaria. Los anticonceptivos hormonales inyectables son efectivos en casos de dificultad en la adherencia diaria al tratamiento oral, sin embargo pueden presentar aumento de peso y pérdida significativa de densidad ósea. el uso en la pubertad es discutible. Los implantes subdérmicos de etonogestrel están indicados para reducir la dismenorrea y en casos susceptibles de tromboembolismo venoso. Los anticonceptivos de barrera son eficaces para prevenir el embarazo y, algunas, incluso las infecciones de transmisión sexual. Consideraciones finales: Cada paciente debe ser evaluada de acuerdo con las particularidades clínicas, sociales, personales y psicológicas, siendo la elección del método anticonceptivo entre médico y paciente. Es necesario exponer los riesgos y beneficios del método elegido y orientar el uso asociado al anticonceptivo de barrera.

Palabras clave: Adolescente, Anticonceptivos, Cumplimiento de la medicación.

\section{INTRODUÇÃO}

Durante a adolescência surgem inúmeras modificações físicas, cognitivas, emocionais e comportamentais. Nesta ocorrem buscas por novas experiências, o que pode incluir uso de álcool ou outras drogas e atividade sexual, muitas vezes desprotegida, com riscos a saúde sexual e reprodutiva, tais como gravidez indesejada e infecções sexualmente transmissíveis (ISTs) (TODD N, et al., 2020). Diferentes estudos revelam que adolescentes iniciam práticas sexuais cada vez mais cedo e isso, não raro, associa-se a múltiplos parceiros, altas taxas de ISTs, maiores índices de aborto e complicações gestacionais (MIRANDA PSF, et al., 2018).

Por outro lado, normalmente a menarca ocorre dos 12 aos 13 anos, o que, fisiologicamente, permite às adolescentes, sexualmente ativas, engravidarem. Diante do fato de haver muitas indicações não contraceptivas para uso de anticoncepcionais hormonais, como menorragia, dismenorréia, síndrome prémenstrual, síndrome dos ovários policísticos, endometriose e adenomiose, muitas adolescentes abaixo dos 15 anos usam métodos contraceptivos hormonais $(\mathrm{MCH})$, mesmo sem ainda ter tido relação sexual (WOODS, G, et al., 2016). Contudo, segundo dados da Secretaria de Atenção Primária à Saúde (SAPS), divulgados pelo Ministério da Saúde (MS), de 2020, no Brasil, cerca de 930 adolescentes e jovens dão à luz diariamente, totalizando mais de 434,5 mil por ano.

A taxa mundial de gravidez na adolescência é estimada em 46 nascimentos para cada mil mulheres entre 15 e 19 anos. Na América Latina e no Caribe, a taxa é estimada em 65,5 nascimentos, enquanto, no Brasil, chega a 68,4. Globalmente, o risco de morte materna é duplicado em mães, com menos de 15 anos, em países de baixa e média renda, e as mortes perinatais estão $50 \%$ acima quando elas possuem menos de 20 anos, se comparadas a nascidos de mães entre 20 e 29 anos (BRASIL, 2020).

No Brasil, a Lei no 13.789, de 3 de janeiro de 2019, instituiu a Semana Nacional de Prevenção da Gravidez na Adolescência, a ser realizada anualmente na primeira semana de fevereiro visando promover informações preventivas e educativas que auxiliem a reduzir casos de gravidez na adolescência. Entre os métodos contraceptivos existentes, no Brasil, estão hormonais e não hormonais, reversíveis e irreversíveis. Entre os hormonais existem compostos apenas por progestágenos e compostos por estrógenos e progestágenos. Os não hormonais incluem métodos de barreira, comportamentais e mecânicos. Todos são tidos como reversíveis. Entre os irreversíveis estão a laqueadura tubária e a vasectomia (SOCIEDADE BRASILEIRA DE PEDIATRIA (SBP), 2018; FEDERAÇÃO BRASILEIRA DAS ASSOCIAÇÕES DE GINECOLOGIA E OBSTETRÍCIA (FEBRASGO, 2015). Destes, nove estão disponíveis no Sistema Único de Saúde (SUS) (BRASIL, 2020).

Mesmo diante de tantos métodos disponíveis, ainda se observa um tabu quanto a informar adolescentes sobre estes. Estudos demonstram que, diferentemente do que se pensa, isso não estimula vida sexual precoce, mas prepara para decisões conscientes e responsáveis sobre o corpo (BRASIL, 2020). Assim, 
discutir esse tema com adolescentes é fundamental, por se tratar de uma questão de saúde pública, afinal, lida com promoção da saúde do adolescente e prevenção de doenças e complicações decorrentes de gestações indesejadas ou ISTs (SILVA AF, 2018). Diante disso, a Organização Mundial da Saúde (OMS) reforça que nenhum método contraceptivo é contraindicado com base apenas na idade (OMS, 2015).

A adesão também é um fator relevante, ao passo que muitas adolescentes podem temer o ganho de peso, alterações no padrão de sangramento, acne, alterações do humor, dentre outros efeitos colaterais desses métodos (TODD N, et al., 2020). Quanto aos riscos da contracepção hormonal na adolescência, o tromboembolismo venoso (TEV) é uma complicação rara, porém potencialmente fatal, do uso dos métodos hormonais combinados, sendo esse risco maior no primeiro ano de uso do método, particularmente, nos primeiros 6 meses (WOODS G, et al., 2016).

Dessa forma, o objetivo do presente estudo consiste em identificar os fatores relacionados à escolha do método contraceptivo na adolescência, bem como discutir sobre os riscos e os benefícios de diferentes métodos contraceptivos e as indicações destes em adolescentes e mulheres jovens.

\section{REVISÃO BIBLIOGRÁFICA}

A adolescência e a juventude são definidas pela OMS como período dos 10 aos 19 anos e dos 15 aos 24 , respectivamente (TODD N, et al., 2020). Estudo realizado por TOOD N et al, 2020, mostrou que a maior parte dos países ocidentais, a sexarca ocorre em torno dos 17 anos, de modo que aos 20 , quase $80 \%$ das mulheres já tiveram relação sexual e, muitas, com mais de um parceiro. Outro estudo realizado na UNICAMP, em 2018, com questionário para 1.193 adolescentes homens e mulheres, mostrou que $41 \%$ iniciaram a vida sexual entre 13 e 15 anos (SILVA AF, 2018).

Dados do relatório conjunto da Organização Pan-Americana da Saúde/Organização Mundial da Saúde (OPAS/OMS), Fundo das Nações Unidas para a Infância (UNICEF) e Fundo de População das Nações Unidas (UNFPA), no Brasil, aproximadamente 930 adolescentes e jovens têm filhos todos os dias, em um total próximo de 434,5 mil mães adolescentes por ano. Embora este número esteja em queda, mesmo assim o Brasil ainda registra a maior taxa entre os países da América Latina e Caribe, chegando a 68,4 nascidos vivos para cada mil adolescentes e jovens (BRASIL, 2020).

Estudo do MS, chamado Saúde Brasil, indica altas taxas de mortalidade infantil associadas a mães até 19 anos, com 15,3 óbitos para cada mil nascidos vivos, sendo a taxa nacional de 13,4. Esses resultados obstétricos são possivelmente influenciados por imaturidade biológica e condições socioeconômicas desfavoráveis (BRASIL, 2019).

Assim, fica clara a importância de saber e divulgar adequadamente métodos contraceptivos existentes, bem como os disponíveis no SUS. Entre os existentes no Brasil estão hormonais e não hormonais, e reversíveis, e irreversíveis. Entre os hormonais existem compostos apenas por progestágenos, como implantes subdérmico de etonogestrel, Dispositivos Intrauterinos (DIUs) de levonorgestrel, injeções intramusculares de medroxiprogesterona, minipílulas de desogestrel e dienogeste, e compostos por estrógenos e progestágenos, como pílulas orais, anéis vaginais, adesivos transdérmicos e injeções mensais (FEBRASGO, 2015).

Os não hormonais incluem métodos comportamentais, como lactação/amamentação, curva de temperatura basal, avaliação do muco cervical, tabela de Ogino-Knauss, sintotérmico e coito interrompido; métodos de barreira, como preservativos feminino e masculino, diafragma e espermicida; e mecânicos, como DIUs de cobre ou cobre com prata. Estes são tidos como reversíveis. O método irreversível feminino é a laqueadura tubária. É importante frisar que alguns métodos, conhecidos por Long-Acting Reversible Contraception (LARC), são classificados como de longa duração, considerados os mais seguros do mercado, deles fazem parte DIUs de levonorgestrel e implantes subdérmicos de etonogestrel (FEBRASGO, 2015).

O índice de Pearl, que é o índice que avalia o número de gestações que ocorrem durante o primeiro ano de uso do método anticoncepcional para cada 100 mulheres, leva em consideração o uso típico e o uso perfeito do método. Diante dessa análise os LARCs e a laqueadura são tidos como os métodos mais seguros 
por apresentarem um risco menor do que o de uma gestação por 100 mulheres após um ano de uso (SBP, 2018; FEBRASGO,2015). Desses métodos, nove estão disponíveis no SUS, podendo ser utilizado por adolescentes, que são preservativo feminino e masculino, pílulas combinados e com progestágeno isolado, DIU de cobre, Diafragma, Pílula do dia seguinte, e Anticoncepcionais injetáveis mensais e trimestrais (BRASIL, 2020)

É indiscutível a importância do uso de contraceptivos em adolescentes, visto que o uso está relacionado à diminuição substancial da gravidez na adolescência nos últimos 25 anos (CHANDRA-MOULO V e AKWARA $E, 2020)$. Entretanto, o uso de contracepção hormonal está associado a riscos que devem ser ponderados (CARDOSO LCDS, et al., 2019).

\section{Contraceptivos Hormonais Orais}

Existem no mercado diversos métodos contraceptivos reversíveis utilizados por adolescentes que podem oferecer riscos quando indicados inadequadamente, como os hormonais orais que podem apresentar-se apenas como progestágenos isolados ou na forma conjugada estrogênio e progestogênio, podendo ser classificados em monofásicos, bifásicos e trifásicos (CARDOSO LCDS, et al., 2019). Os monofásicos, considerados os mais utilizados, possuem mesma composição e dosagem hormonal em todos comprimidos; os bifásicos apresentam composição idêntica, porém duas doses diferentes de estrogênios e progestogênios; e os trifásicos exibem três dosagens distintas (FEBRASGO, 2015).

Os contraceptivos reversíveis mais utilizados são os Anticoncepcionais Conjugados Orais (ACOs), que combinam formas sintéticas dos hormônios estrogênio e progestogênio, semelhantes aos produzidos pelos ovários. Entretanto, esses hormônios podem induzir alterações no comportamento socioemocional e na função cerebral (MONTOYA ER e BOS PA, 2017). Existem evidências na literatura que hormônios esteroides influenciam regiões corticais e subcorticais, elevando riscos de depressão na vida adulta em mulheres que utilizaram anticoncepcional oral na adolescência (ANDERL C, et al., 2019).

Concomitantemente, a utilização de ACOs também está associada a Trombose Venosa Profunda (TEV), na qual ocorre a formação de coágulo em veias profundas, obstruindo vasos parcial ou totalmente (WOODS GM, et al., 2016). Além do TEV, pode ocorrer, ainda, a trombose venosa cerebral (TVC), que consiste na formação de coágulos em veias e seios venosos cerebrais. Essa condição clínica é rara e muitas vezes subdiagnosticada (OLIVEIRA LFD, et al., 2016; ÖZDEMIR HH, et al., 2015).

Ademais, um estudo observacional prospectivo, realizado no Canadá, com 527 mulheres entre 16 a 24 anos, comparou o uso de anticoncepcionais hormonais combinados e mudanças na Densidade Mineral Óssea (DMO) por 2 anos. Os autores verificaram que as usuárias apresentaram menor acúmulo de DMO no colo do útero e na região do quadril quando comparadas a não usuárias (BRAJIC, et al., 2018).

Apesar dos riscos associados, o uso do ACO, quando indicado corretamente, oferece benefícios acerca de proteção em casos de anemia, cistos foliculares, doença inflamatória pélvica (DIP), diminuição dos sintomas da dismenorreia, dos ciclos hiperhemorrágicos e da endometriose. Eles auxiliam, ainda, na regulação do ciclo menstrual, diminuem cólicas e oleosidade excessiva da pele, melhorando, assim, quadros de acne (TODD N e BLACK A, 2019).

A acne vulgar é considerada uma doença inflamatória da pele, multifatorial, muito comum em mulheres jovens, porém os andrógenos desempenham um papel importante na produção de sebo, principalmente em adolescentes (PICARDO M, et al., 2017). O tratamento geralmente depende do grau de acometimento. ACOs propiciam resultados positivos no trato de adolescentes, pois atuam inibindo a secreção do FSH e do LH por feedback negativo na hipófise, reduzindo a produção de androgênio ovariano. O estrogênio também favorece a ligação de globulinas a testosterona livre circulante, reduzindo os androgênicos séricos (NGUYEN HL e TOLLEFSON MM, 2017).

É importante destacar que taxas de sucesso do ACOs dependem da ingestão diária, o que pode elevar a taxa de falha de $0,01 \%$ para aproximadamente $8 \%$ quando administrado incorretamente. A indicação é bastante difundida, especialmente devido à alta acessibilidade e baixo custo em comparação a outros 
métodos como os LARC. Recomenda-se que, durante a seleção deste, sejam avaliados a dose de estrógeno, o tipo de progestágeno e o custo do fármaco, estabelecendo melhor relação custo-benefício, respeitando critérios de elegibilidade para cada adolescente (LUBIANCA JN, 2016).

Os anticoncepcionais que apresentam formulação com apenas progestágenos isolados são recomendados para lactentes e com contraindicação ao uso de estrógeno, como no caso de mulheres com riscos de trombose arterial ou embolia pulmonar. Progestágenos não afetam significativamente a coagulação nem o risco de acidente vascular encefálico, desde que inexistam outros fatores (GIGLIO MRP, et al., 2015). Entretanto, no caso do uso de progestágeno isolado pela via oral, exige-se maior precisão relacionada aos horários de consumo, pois a efetividade reduz quando se esquece de ingerir ou atrasa-se (CARDOSO LCDS, et al., 2019)

\section{Dispositivo intrauterino (DIU)}

Discute-se muito acerca da utilização do DIU como contraceptivo em adolescentes e nulíparas. Ele exerce ações locais e apresenta-se de duas maneiras: não hormonal (constituído de cobre ou cobre e prata) e hormonal (liberador de levonorgestrel). A possível relação do uso com o aumento de taxas de DIP ou mesmo o risco de perfuração uterina, levou, diversas vezes, à restrição para adolescentes (PATSEADOU $M$ e MICHALA L, 2017).

Um estudo de coorte prospectivo recente, realizado pelo Contraceptive CHOICE Project, avaliou 9000 mulheres, entre 14 e 45 anos, para observar o aumento de DIP em usuárias de DIU. Elas foram acompanhadas por até 3 anos após a inserção e concluiu-se que usuárias do dispositivo, em comparação com usuárias de outros LARC, não apresentaram aumento nas taxas da doença. Logo, considerou-se o DIU um método seguro, inclusive para adolescentes (SECURA GM, et al., 2010).

Já o Estudo Europeu de Vigilância Ativa em Dispositivos Intrauterinos observou 61.648 mulheres, de seis países europeus, visando correlacionar o risco de perfuração uterina e a inserção do DIU. Destas, 81 relataram perfuração e, a maioria deles, em associação com amamentação no momento da inserção, qualificando assim um baixo risco de complicação por perfuração e sem diferenças clínicas entre DIU e SIULNG (HEINEMANN K, et al., 2015).

Estudos dirigidos pela American Academy of Pediatrics e American College of Obstetricians and Gynecologists mostraram taxa de sucesso de $96 \%$ na primeira tentativa de colocação do DIU em adolescentes, com falha e expulsão não correlacionadas à idade, tipo ou mesmo paridade (ESPEY E, et al., 2019).

Ao contrário do DIU não hormonal, que pode alterar o padrão menstrual ocasionando aumento do fluxo, o hormonal pode agir de maneira a reduzi-lo. O DIU de cobre é um método seguro, já que dispensa intervenção diária, com efetividade de $99,3 \%$, e caracteriza uma boa opção para quem apresenta aversão a métodos hormonais. O DIU liberador de levonorgestrel segue a mesma linha de eficácia do não hormonal, com padrão de efetividade de $99,8 \%$, sendo passível de interrupção no momento que a usuária desejar, assim como o não hormonal. Entretanto, apesar de ambos serem muito efetivos, deve-se recomendar a associação deles a preservativos, visto que adolescentes são grupos de risco para ISTs (BRASIL, 2018).

Anteriormente, adolescentes eram consideradas inelegíveis para uso de DIUs, tendo como justificativa maior susceptibilidade às ISTs, preocupações com fertilidade a longo prazo e possível dificuldade na inserção. Entretanto, nos Critérios de Elegibilidade Médica para Uso de Contraceptivos da OMS, de 2015, o DIU Liberador de Levonorgestrel é considerado categoria 2 para mulheres com menos de 20 anos, principalmente pelas preocupações com expulsão e ISTs. Apesar disso os benefícios, geralmente, superam riscos associados ao uso (PATSEADOU M e MICHALA, L, 2017; OMS, 2015).

\section{Anticoncepcionais hormonais injetáveis}

Os anticoncepcionais hormonais injetáveis de administração mensal seguem os mesmos critérios de indicações e contraindicações dos ACOs, sendo três formulações disponíveis no Brasil: o acetato de 
Medroxiprogesterona 25mg associado ao Cipionato de Estradiol 5mg; Enantato de Noretisterona 50mg associado ao Valerato de Estradiol 5mg; e Algesterona Acetofenida 150mg associado ao Enantado de Estradiol 10mg.

O Acetato de Medroxiprogesterona de Depósito (DMPA) é uma das formas de contracepção reversível mais difundidas mundialmente devido à alta eficácia. Ele é composto por progestágeno, administrado trimestralmente; atua suprimindo a ovulação, logo, além dos benefícios contraceptivos, também é utilizado em tratamentos de dismenorreia e endometriose, concomitantemente, apresenta efeito protetor contra 0 câncer endometrial. Segundo a OMS, o DMPA possui categoria 2 no critério de elegibilidade em menores de 18 anos e categoria 1 para mulheres com idade entre 18-45 anos (LUBIANCA JN, 2016; SWEENEY B, et al., 2020). Estudos demonstram que grande parte das usuárias fazem interrupção do tratamento com DMPA ainda no primeiro ano devido a efeitos colaterais, em especial, aumento significativo de massa corporal (FARIAS AGS, et al., 2017; SWEENEY B, et al., 2020).

Um comparativo acerca da redução de massa óssea em usuárias de DMPA obteve um percentual de $3,17 \%$ a mais que em usuárias de ACOs. Apesar dos efeitos desfavoráveis sobre a densidade de massa óssea ao longo do uso na adolescência, há uma tendência de recuperação após descontinuação (LUBIANCA JN, 2016). O método apresenta alta taxa de eficiência, os contraceptivos injetáveis apresentaram taxa de gravidez entre 0 a 0,7/100 mulheres por ano (BRANDT GP, et al., 2018).

O uso dos anticoncepcionais injetáveis é eficiente para quem possui dificuldades em utilizar contracepção oral diariamente. Para pacientes jovens, o risco de eventuais esquecimentos na administração diária deve ser considerado durante a escolha, de maneira que, pela administração dos injetáveis apresentar dependência reduzida da usuária, haja maior sucesso contraceptivo (LUBIANCA JN, 2016).

\section{Implantes subdérmicos de etonogestrel}

Implantes subdérmicos de etonogestrel apresentam alta taxa de eficácia entre os LARC, com taxa de 99,9\% de eficiência, sendo superior a procedimentos definitivos como a laqueadura. (ROMANO ME, et al., 2019). Esse método consiste em um sistema de liberação hormonal alternativo à via oral, contornando o metabolismo de primeira passagem hepática. Ademais, tem-se vantagem de liberação mais constante, lenta e prolongada dos esteroides. O bastão de etonogestrel contém por volta de $68 \mathrm{mg}$ do metabólito ativo de desogestrel, apresentando quantidade de liberação decrescente ao longo dos três anos de duração (LUBIANCA JN, 2016).

De modo geral, o principal mecanismo de ação dos implantes é a disfunção ovulatória. Isso propicia alterações histológicas e na densidade endometrial, levando à redução da espessura da parede endometrial. O efeito progestogênico aumenta a aderência do muco cervical, inibindo a inserção dos espermatozoides. Além do benefício anticoncepcional, o implante apresenta ampla indicação para redução da dismenorreia e é considerado seguro em pacientes suscetíveis ao desenvolvimento de tromboembolismo venoso (LUBIANCA JN, 2016).

Em contrapartida, a imprevisibilidade menstrual foi relatada como principal efeito colateral dos implantes; escapes menstruais estão entre as queixas mais comuns, podendo levar a descontinuação (LUBIANCA JN, 2016). Poucos são os estudos que analisaram o efeito do implante em relação à DMO, já que métodos contraceptivos que utilizam apenas progestagênio por uso prolongado podem causar alterações na aquisição óssea e induzir à perda precoce, oferecendo riscos de osteoporose.

Um estudo prospectivo realizado na Unidade de Reprodução Humana da Faculdade de Ciências Médicas da Universidade Estadual de Campinas, Brasil, avaliou e comparou, por até 12 meses, 75 mulheres hígidas adeptas ao DIU de cobre e implante de etonogestrel. Concluiu-se que usuárias de ambos os métodos não apresentaram diferenças no que diz respeito à DMO. Assim, mesmo as jovens que ainda não atingiram o pico de massa óssea, podem usar o implante subdérmico despreocupadas em relação à DMO (MODESTO W, et al., 2015).

Um estudo feito através do subgrupo de mulheres inscritas no Projeto $\mathrm{CHOICE}$, com base no relato de ganho de peso pelas usuárias de MCLD, comparou de maneira prospectiva 149 participantes sem 
manifestações metabólicas ou alterações de $5 \%$ no peso corporal no último semestre. As participantes, com idade média de 25,9 anos, usuárias de DIU de cobre, Sistema Intrauterino de Levonorgestrel (SIU-LNG) e Implante de Etonogestrel (implant ENG) foram analisadas e concluiu-se que alterações no peso corporal após 12 meses não estavam associadas a um tipo específico de método, não podendo ser listadas como efeito colateral direto (SANTOS PNS, et al., 2017).

De acordo com os critérios de elegibilidade da OMS, os implant ENG são classificados em categoria 1 para mulheres a partir da menarca até 18 anos. Ademais, devido à independência da usuária e ao êxito contraceptivo de longa duração, pode ser considerado uma alternativa assertiva para adolescentes (LUBIANCA JN, 2016).

\section{CONSIDERAÇÕES FINAIS}

A orientação contraceptiva e o seu uso na adolescência são de extrema importância para a saúde física, sexual e mental dessa população. Tendo em vista que a inutilização ou o uso inadequado de métodos contraceptivos podem levar à aquisição de ISTs e a uma gravidez não planejada na adolescência, é importante que a escolha do método ideal seja feita pelo médico e paciente, em conjunto. Os métodos de longa duração mostram-se superiores em eficiência por não dependerem da administração diária pela adolescente. No entanto, é imprescindível avaliar a paciente de maneira individualizada, considerando suas particularidades clínicas, sociais, pessoais e psicológicas, bem como expor os riscos e os benefícios de cada método contraceptivo e sanar todas as dúvidas. Ademais, deve-se reforçar quanto ao uso de preservativos no ato sexual, evidenciando ser o único método capaz de prevenir ISTs.

\section{REFERÊNCIAS}

1. ANDER C, et al. Oral contraceptive use in adolescence predicts lasting vulnerability to depression in adulthood. J. Child Psychol. Psychiatry, 2019; 61(2): 148-156.

2. BRAJIC TS, et al. Combined hormonal contraceptives use and bone mineral density changes in adolescent and young women in a prospective population based Canada-wide observational study. J Musculoskelet Neuronal Interact, 2018; 18(2):227-236.

3. BRANDT GP, et al. Anticoncepcionais hormonais na atualidade: um novo paradigma para o planejamento familiar. Revista Gestão \& Saúde. RGS, 2018; 18(1): 54-62.

4. BRASIL. Lei № 13.798, de 3 de Janeiro de 2019. Brasília, Disponível em: http://www.planalto.gov.br/ccivil_03/_ato2019-2022/2019/lei/L13798.htm. Acesso em: 10 abr. 2021.

5. BRASIL. Ministério da Saúde. Manual Técnico para Profissionais de Saúde: DIU com Cobre TCu 380A. Brasília, 2018.

6. BRASIL. Ministério da Saúde. Nota Técnica No 1/2020-COSAJ/CGCIVI/DAPES/SAPS/MS: Semana Nacional de Prevenção da Gravidez na Adolescência. 2020. Disponível em: https://egestorab.saude.gov.br/image/?file=20200206_N_NTcampanhagravideznaadolescencia_7488128670569364 322.pdf. Acesso em: 10 abr. 2021

7. BRASIL. Ministério da Saúde. Secretaria de Atenção Primária à Saúde. Departamento de Ações Programáticas Estratégicas. Coordenação-Geral de Ciclos da Vida. Coordenação de Saúde dos Adolescentes e Jovens. Brasília, 2020.

8. BRASIL. Ministério da Saúde. Secretaria de Atenção à Saúde. Departamento de Ações Programáticas Estratégicas. Direitos Sexuais, Direitos Reprodutivos e Métodos Anticoncepcionais. Brasília, 2006.

9. BRASIL. Ministério da Saúde. Uma análise da situação de saúde e das doenças e agravos crônicos: desafios e perspectivas. Saúde Brasil. Brasília, 2019.

10. CARDOSO LCDS, et al. A utilização de contraceptivos hormonais por adolescentes e potenciais riscos para a saúde. Clin Biomed Res., 2019; 39(1): 75-84.

11. CHANDRA-MOULI V, AKWARA E. Improving access to and use of contraception by adolescents: What progress has been made, what lessons have been learnt, and what are the implications for action? Best Pract Res Clin Obstet Gynaecol., 2020; 66: 107-118.

12. ESPEY E, et al. Barriers and solutions to improve adolescent intrauterine device access. J Pediatr Adolesc Gynecol., 2019; 32(5): S7-S13.

13. FARIAS AGS, et al. Satisfaction of combined and exclusive injectable contraceptive users of progestogen and associated factors. Revista Rene, 2017; 18(3): 345-52.

14. FEDERAÇÃO BRASILEIRA DAS ASSOCIAÇÕES DE GINECOLOGIA E OBSTETRÍCIA (FEBRASGO). Manual de Anticoncepção, 2015.

15. FEDERAÇÃ̉O BRASILEIRA DAS ASSOCIAÇÕES DE GINECOLOGIA E OBSTETRÍCIA (FEBRASGO). Tratado de ginecologia. Satisfaction of combined and exclusive injectable contraceptive users of progestogen and associated factors. Rio de Janeiro: Elsevier, 2019. 
16. FERRI FF. Ferri ginecologia e obstetrícia. 1. ed. Rio de Janeiro: Elsevier, 2019.

17. GIGLIO MRP, et al. Contracepção hormonal segundo a ótica do estudante de medicina: Mais um desafio para o ensino médico brasileiro?. Rev. Bras. de Educação Médica, 2015; 39(4): 502-506.

18. HEINEMANN K, et al. Risk of uterine perforation with levonorgestrel-releasing and copper intrauterine devices in the European Active Surveillance Study on Intrauterine Devices. Contraception, 2015; 91(4): 274.

19. HOFFMAN BL, et al. Ginecologia de Williams. 2. ed. Porto Alegre, Artmed, 2014.

20. LANNES DRC, GONZAGA LL. "Rejeição Do Uso de Camisinha Por Adolescentes: Uma Perspectiva a Partir Da Zona Muda Das Representações Sociais." Revista Espaço Pedagógico, vol. 25, no. 2, 472-487. 2018.

21. LUBIANCA JN. Opções de anticoncepção na adolescência. Uso Racional de medicamentos: fundamentação em condutas terapêuticas e nos macroprocessos da Assistência Farmacêutica. Brasília, 2016.

22. MIRANDA PSF, et al. Sexual behaviors: study in the youth. Einstein (São Paulo), São Paulo, 2018; 16(3): 1-7.

23. MODESTO W, et al. Body composition and bone mineral density in users of the etonogestrel-releasing contraceptive implant. Arch Gynecol Obstet., 2015; 292(6): 1387-91.

24. MONTOYA ER, BOS PA. How Oral Contraceptives Impact Social-Emotional Behavior and Brain Function. Trends in Cognitive Sciences, 2017; 21(2): 125-136.

25. NGUYEN HL, TOLLEFSON MM. Endocrine disorders and hormonal therapy for adolescent acne. Curr Opin Pediatr., $2017 ; 29(4): 455-65$.

26. OLIVEIRA LFD, et al. Trombose venosa cerebral e alterações cognitivas. Revista Brasileira de Neurologia e Psiquiatria, 2016; 20(1): 107-113.

27. ÖZDEMIR HH, et al. Evaluation of cerebral venous thrombosis secondary to oral contraceptive use in adolescents. Neurological Sciences, 2015; 36(1): 149-153.

28. PATSEADOU M, MICHALA L. Usage of the levonorgestrel-releasing intrauterine system (LNG-IUS) in adolescence: what is the evidence so far? Arch Gynecol Obstet., 2017; 295(3): 529-541.

29. PICARDO M, et al. Acne and rosacea. Dermatol Ther. 2017; 7(1): 43-52.

30. ROMANO ME, et al. Assessing weight status in adolescent and young adult users of the etonogestrel contraceptive implant. Journal of pediatric and adolescent gynecology, 2019; 32(4): 409-414.

31. SANTOS PNS, et al. Changes in body composition in women using long-acting reversible contraception. Contraception, 2017; 95(4): 382-389.

32. SECURA GM, et al. The Contraceptive CHOICE Project: reducing barriers to long-acting reversible contraception. Am J Obstet Gynecol. 2010; 203(2): 115.e1-115.e1157.

33. SILVA AF, LOPES MHBM. Uso de métodos anticoncepcionais entre adolescentes de ensino médio. Adolesc Saude, 2018; 15(2): 102-112.

34. SOCIEDADE BRASILEIRA DE PEDIATRIA (SBP). Anticoncepção na Adolescência. Guia Prático de Atualização, 2018; 7(2): 1-16.

35. SWEENEY B, et al. Clinical approaches to minimize iatrogenic weight gain in children and adolescents. Clinical Obesity, 2020; 11(1): 1-5.

36. TODD N, BLACK A. Contraception for Adolescents. J Clin Research in Pediatric Endocrinology, 2020; 12(Suppl 1): 2840.

37. WOODS GM, et al. A Review of Hormonal Contraception and Venous Thromboembolism in Adolescents. J Pediatr Adolesc Gynecol, 2016; 29(5): 402-408.

38. WORLD HEALTH ORGANIZATION (WHO). Medical Eligibility Criteria for Contraceptive use, 2015. 